\title{
PARTIAL ANOMALOUS PULMONARY VENOUS DRAINAGE IN ASSOCIATION WITH ATRIAL SEPTAL DEFECT
}

\author{
BY
}

\author{
M. S. GOTSMAN*, R. ASTLEY, AND C. G. PARSONS \\ From The Children's Hospital, Birmingham 16 \\ Received August 6, 1964
}

Partial anomalous pulmonary venous drainage occurs in about 10 per cent of patients with atrial septal defect, whether this be of a sinus venosus type, a centrally situated secundum defect, or a large posterior defect with no posterior margin (pseudo-anomalous right pulmonary veins) (Table I). The surgical anatomy of the sinus venosus defect was first described in detail by Lewis et al., (1955) who recognized the absence of a posterior rim and upper margin and the association with partial anomalous pulmonary venous drainage. It was important to recognize the condition before operation since it made repair difficult, and cardiopulmonary bypass was always required to achieve a satisfactory result (Weidman et al., 1957; Bedford et al., 1957; Wood, Conrad, and Morrow, 1957; Bahnson, Spencer, and Neill, 1958; Morrow et al., 1960; LePere, Thomson, and Kohler, 1962; Lawrance et al., 1962).

TABLE I

Incidence of Partial Anomalous Pulmonary Venous Drainage (PAPVD)

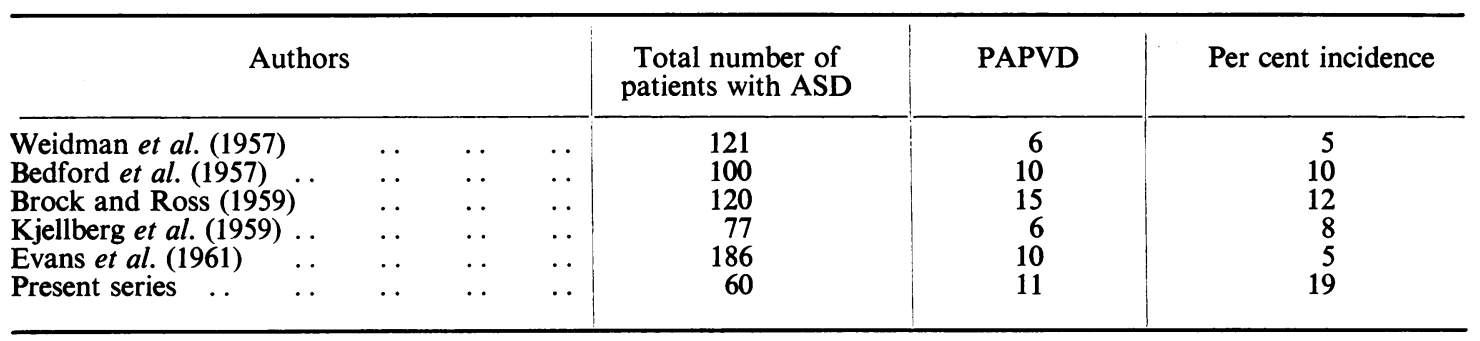

\section{SubjeCtS AND MeTHODS}

Eleven children were seen with this defect. The diagnosis was made by cardiac catheterization in 9 and confirmed by operation in 8 . In 2 other children, catheterization showed only an atrial septal defect and the anomalous veins were not discovered until operation. One of these children died, for hypothermia only was used and a prolonged period of circulatory arrest was required. In one additional child a false positive diagnosis of an anomalous vein was made.

Cardiac catheterization was carried out in all subjects, the catheter being passed from the elbow or the groin, depending on the size of the patient and the availability of suitable veins. Oxygen saturation in blood samples was measured with a Kipp C.C. oximeter. This causes such a small loss of blood that it was possible, even in small children, to take duplicate samples from the right atrium at three levels-high, mid,

* Present address: Cardiac Clinic, Groote Schuur Hospital, Observatory, Cape Town, South Africa. 
and low-and from three points $1 \mathrm{~cm}$. apart at the lower end of the superior vena cava. The atrium was explored methodically in an attempt to introduce the catheter tip into a pulmonary vein orifice, and the position of the catheter was determined by careful screening, using an image intensifier. When a pulmonary vein was entered, angiocardiograms were taken to define its position more precisely. On two occasions shunts were confirmed by a catheter incorporating a platinum black electrode which was activated by inhaled hydrogen.

\section{RESULTS}

Clinical Features. The clinical features and electrocardiograms were identical with those found in secundum defects of the atrial septum, though 5 patients had tall peaked $P$ waves. In no case was a diagnosis made until cardiac catheterization, though Dow (1959) suggests that the anomaly should be suspected when radiography of the chest or tomograms of the right superior atrio-caval junction reveal narrowing of the superior vena cava in its upper part, a bulge in its lowest centimetre, or a high insertion of pulmonary veins from the upper lobe of the right lung.

Hamodynamics. Details of the patients and the hæmodynamic findings are given in Table II. The mean pressure difference between the pulmonary artery and the left atrium was $14.5 \mathrm{~mm} . \mathrm{Hg}$ if two patients with associated pulmonary stenosis are excluded. In a comparable series of 40 patients with simple secundum atrial septal defect who were studied during the same period the pressure difference was $9 \cdot 1 \mathrm{~mm}$. Hg. The difference between the two means is highly significant $(t=7 \cdot 55, p<0.001)$. This confirms the impression of Evans, Rowe, and Keith (1961). However, the range of values is too great to allow this fact to be of practical value in diagnosis.

Oxygen Saturation. Five patients had serial samples taken from the junction of the superior vena cava with the right atrium, and in all there was a rise of oxygen saturation either low in the vena cava or high in the right atrium. However, evidence that this was due to anomalous pulmonary venous drainage was conclusive only in two patients. Brotmacher and Fleming (1957) suggested that in the superior vena cava a rise in oxygen content of more than $2.2 \mathrm{ml}$. per cent or a saturation exceeding 88 per cent is diagnostic of anomalous drainage into the superior vena cava. We have found that multiple samples taken from the superior vena cava of children with simple atrial septal defects may show considerable variations in oxygen saturation, and we are consequently apt to disregard such findings unless the differences are large. On this account we failed to recognize an anomalous pulmonary vein in one of our patients though we had demonstrated a small difference in oxygen saturation (Weidman et al., 1957).

Indicator Dilution Studies. The hydrogen circulation time was short in two patients with anomalous venous drainage into the superior vena cava. The finding is not specific because, even when there are no anomalous veins, a large shunt through an atrial septal defect may be associated with atrio-caval reflux and consequently a shortened circulation time. We have not used other indicators in these patients (Swan et al., 1957; Lawrance et al., 1962).

Position of the Catheter. The catheter was introduced into the mouth of the anomalous vein(s) in all 9 patients who were correctly diagnosed at catheterization. The catheter failed to enter the anomalous vein in 2 , and in neither of these was the condition recognized before operation. Wood (1956) considered that a catheter entering the right superior pulmonary vein was diagnostic, but the finding is not specific, for the catheter may pass through an atrial septal defect or a valved foramen ovale and enter a pulmonary vein which drains normally into the left atrium. If a catheter, passed from the arm, enters what appears to be an anomalous vein well above the border of the cardiac shadow, passage across the atrial septum can be excluded and the diagnosis may be regarded as established. If, however, the vein is entered at a lower level the diagnosis is less certain.

The atrial septum lies at an angle of about $45^{\circ}$ to the sagittal plane with the left atrium lying postero-laterally (Walmsley, 1958). The common practice during cardiac catheterization is to screen the patient only in the postero-anterior position in which the two atria are superimposed (Fig. 1), and it may not be obvious that the catheter has passed backwards into the left atrium through a septal defect. This is particularly likely to happen if the defect has a narrow right rim or, in the 
TABLE $\stackrel{\mathbb{N}}{=}$

Hamodynamic, Angiocardiographic, and Operative Findings in Patients withe

\begin{tabular}{|c|c|c|c|c|c|c|c|c|c|c|}
\hline \multirow{2}{*}{$\begin{array}{l}\text { Case } \\
\text { No. }\end{array}$} & \multirow{2}{*}{$\begin{array}{l}\text { Age } \\
\text { (yr.) }\end{array}$} & \multirow{2}{*}{$\begin{array}{c}\text { Diagnosis } \\
\text { made or } \\
\text { confirmed at } \\
\text { operation or } \\
\text { necropsy }\end{array}$} & \multirow{2}{*}{$\begin{array}{c}\text { Site of } \\
\text { insertion } \\
\text { of catheter }\end{array}$} & \multirow{2}{*}{$\begin{array}{l}\text { Anomalous } \\
\text { vein entered }\end{array}$} & \multicolumn{6}{|c|}{ Pressures $(\mathrm{mm} . \mathrm{Hg})$} \\
\hline & & & & & RA & RV & PA & $\begin{array}{c}\text { LA or } \\
\text { PW }\end{array}$ & $\Delta \mathbf{P}$ & BA \\
\hline $\begin{array}{r}1 \\
2 \\
3 \\
4 \\
5 \\
6 \\
7 \\
8 \\
9 \\
10 \\
11\end{array}$ & $\begin{array}{r}13 \\
3 \\
7 \\
7 \\
8 \frac{1}{2} \\
6 \\
8 \\
12 \\
4 \\
11 \\
13\end{array}$ & $\begin{array}{l}\text { Op } \\
\mathrm{Op} \\
\mathrm{Op} \\
\mathrm{Op} \\
\mathrm{Op} \\
\mathrm{Op} \\
\overline{\mathrm{Op}} \\
\mathrm{Op} \\
\overline{\mathrm{Op} / \mathrm{N}}\end{array}$ & $\begin{array}{l}\text { Arm } \\
\text { Leg } \\
\text { Leg } \\
\text { Arm } \\
\text { Arm } \\
\text { Arm } \\
\text { Arm } \\
\text { Arm } \\
\text { Arm } \\
\text { Arm } \\
\text { Leg }\end{array}$ & $\begin{array}{l}\text { No } \\
+ \\
+ \\
\text { No } \\
+ \\
+ \\
+ \\
+ \\
+ \\
+ \\
+\end{array}$ & $\begin{array}{r}10 \\
4 \\
9 \\
8 \\
5 \\
4 \\
8 \\
7 \\
10 \\
7 \\
5\end{array}$ & $\begin{array}{r}50 / 4 \\
80 / 2 \\
36 / 0 \\
40 / 2 \\
42 / 2 \\
29 / 0 \\
100 / 5 \\
35 / 4 \\
65 / 0 \\
34 / 2 \\
50 / 5\end{array}$ & $\begin{array}{l}40 / 20(27) \\
32 / 15(21) \\
34 / 12(20) \\
30 / 10(17) \\
20 / 8(12) \\
22 / 7(12) \\
18 / 10(13) \\
35 / 15(22) \\
65 / 24(38) \\
30 / 12(18) \\
46 / 20(29)\end{array}$ & $\begin{array}{r}5 \\
5 \\
9 \\
12 \\
8 \\
3 \\
5 \\
3 \\
10 \\
8 \\
6\end{array}$ & $\begin{array}{r}22 \\
16 \\
11 \\
5 \\
4 \\
9 \\
8 \\
19 \\
28 \\
10 \\
23\end{array}$ & $\begin{array}{r}110 / 75 \\
105 / 60 \\
110 / 80 \\
100 / 70 \\
95 / 60 \\
90 / 60 \\
110 / 70 \\
120 / 70 \\
110 / 80 \\
90 / 60 \\
90 / 55\end{array}$ \\
\hline
\end{tabular}

sinus venosus variety, no right hand margin at all. By contrast, when the subject is rotated into the left anterior oblique position the atria are separated and the catheter can be seen to be directed posteriorly as it passes from right to left atrium.

If, therefore, a right pulmonary vein is entered and the catheter is seen to be directed posteriorly first in the left anterior oblique position, the vein is probably not anomalous. On the other hand,

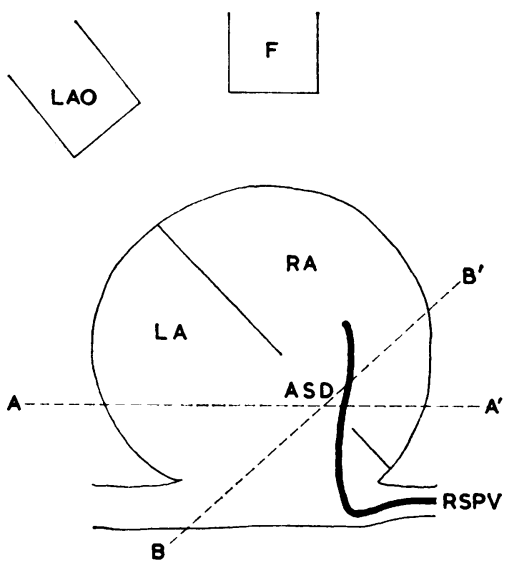

FIG. 1.-The interatrial septum in the left anterior oblique position. The diagram shows a crosssection of the atria in a horizontal transverse plane.

A catheter is shown in place passing through an atrial septal defect or patent foramen ovale (ASD) from the right atrium to the left atrium and then to the right pulmonary vein (RSPV). If the patient is screened in the A-P position (F) the projection $\mathrm{A}-\mathrm{A}$ is seen. The catheter passes backwards and then to the right in this position. It is often impossible to appreciate the backward course taken by the catheter. If the patient is rotated into the left anterior oblique position (LAO) the projection B-B' is seen. This separates the two atria so that the right atrium lies in front of the left atrium. The initial backward course of the catheter is now obvious. if the catheter is directed anteriorly an anomalous insertion is likely.

The exact position of insertion can sometimes be distinguished by withdrawing the catheter very slowly from the pulmonary vein. As the tip of the catheter disengages and lies free in the atrium, it may slip slightly to the side. This may make it possible to determine the atrium into which the vein is emptying.

In the one child in whom a false positive diagnosis was made, the catheter, passed from the arm, entered what appeared to be an anomalous right pulmonary vein. No such vein was found at operation; the mistake was due to failing to screen the patient in the left anterior oblique position.

Angiocardiography. While the position of the catheter as seen in the left anterior oblique projection is a valuable guide to distinguishing between normal and abnormal pulmonary venous drainage, there will be some instances where the exact insertion is still uncertain or the appearances are even frankly misleading. In these cases, we have found angiocardiography the most decisive method of investigation. It was used in 3 of the subjects in the present series, as well as in a number of instances where venous drainage proved to be normal. 
II

Atrial Septal Defect and Partial Anomalous Pulmonary Venous Drainage

\begin{tabular}{|c|c|c|c|c|c|c|c|c|c|}
\hline \multirow{2}{*}{$\begin{array}{l}\text { Pulmonary } \\
\text { systemic } \\
\text { blood flow }\end{array}$} & \multirow{2}{*}{$\begin{array}{l}\text { Hydrogen } \\
\text { electrode }\end{array}$} & \multirow{2}{*}{$\begin{array}{l}\text { Cinefluorography } \\
\text { during rotation } \\
\text { of patient }\end{array}$} & \multicolumn{6}{|c|}{ Selective angiograms } & \multirow{2}{*}{ Findings at Operation } \\
\hline & & & RSPV & SVC & RA & RV & LA & LV & \\
\hline $\begin{array}{r}2 \cdot 8: 1 \\
2: 1 \\
3 \cdot 5: 1 \\
3: 1 \\
5: 1 \\
4: 1 \\
2: 1 \\
2 \cdot 8: 1 \\
2 \cdot 5: 1 \\
2 \cdot 5: 1 \\
2 \cdot 3: 1\end{array}$ & $\begin{array}{l}- \\
\overline{+} \\
+ \\
+ \\
+ \\
+ \\
+ \\
+\end{array}$ & + & $\begin{array}{l}+ \\
+ \\
+\end{array}$ & + & + & $\begin{array}{l}+ \\
+\end{array}$ & $\begin{array}{l}+ \\
+ \\
+\end{array}$ & $\begin{array}{l}+ \\
+ \\
+\end{array}$ & $\begin{array}{l}\text { Sinus venosus; ASD } \\
\text { Large ASD; no posterior rim } \\
\text { Large ASD; no posterior rim } \\
\text { Secundum, ASD } \\
\text { Large ASD; no posterior rim } \\
\text { Sinus venosus, ASD } \\
\text { No operation } \\
\text { Secundum ASD } \\
\text { Secundum ASD } \\
\text { No operation } \\
\text { Sinus venosus ASD }\end{array}$ \\
\hline
\end{tabular}

In the first patient in the present series examined by this method, contrast medium was injected manually while the catheter tip was lying in the anomalous vein. This demonstrated that the vein drained into the right atrium, but injection was slow and the angiocardiogram was of poor quality. Consequently, pressure injections were used and these were much more satisfactory. In the same subject $15 \mathrm{ml}$. of triosil $75 \%$ was injected while the catheter was withdrawn slowly from the anomalous vein with the patient lying in the left anterior oblique position. This was effective in demonstrating the venous insertion but it was difficult to judge the correct speed for withdrawing the catheter which was, in fact, pulled back too rapidly (Fig. 2).

In the next two patients, therefore, the catheter was introduced into the anomalous vein until impaction occurred. It was then withdrawn, for at least $3 \mathrm{~cm}$., until it lay about $2 \mathrm{~cm}$. from the junction of the vein with the superior vena cava. With the patient in the left anterior oblique position, $15 \mathrm{ml}$. of contrast medium was injected and satisfactorily outlined the course of the vein and its drainage into the superior vena cava in one patient and the right atrium in the other (Fig. 3). Selective injection of medium into the right atrium was sufficient to produce reflux filling of an anomalous vein in one patient. In no case was it possible to demonstrate anomalous venous drainage during the lævogram phase of angiocardiography.

In addition, in a number of patients it has been possible to exclude anomalous pulmonary venous drainage, either by injection into a pulmonary vein or into the left atrium (entered via a septal defect or through the foramen ovale). Such atrial injections commonly show the normally inserted veins by reflux into their terminal portions (Fig. 4 and 5).

We have observed no ill effects from pressure injections directly into the pulmonary veins.

Since the introduction of closed circuit television, angiocardiograms have been monitored. The output of the intensifier passes through an image distributor and most of the light is received by the cine camera. However, a small proportion passes to the television camera, allowing the angiocardiogram to be observed. The sequence of events is rapid, but usually enough can be seen to give a provisional diagnosis and to guide subsequent steps in the cardiac catheterization. If the injection has failed to provide the requisite information it can be repeated. This means that complete definitive diagnosis is possible before the catheter is removed.

\section{SUMMARY}

In children with an atrial septal defect, additional partial anomalous pulmonary venous drainage is common and should be recognized before operation since cardiopulmonary bypass is required for 


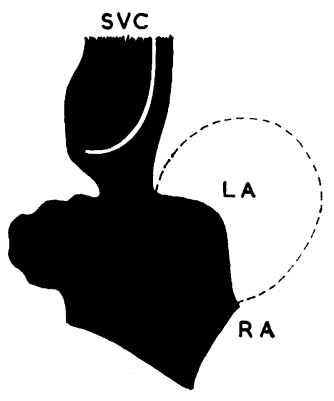

2

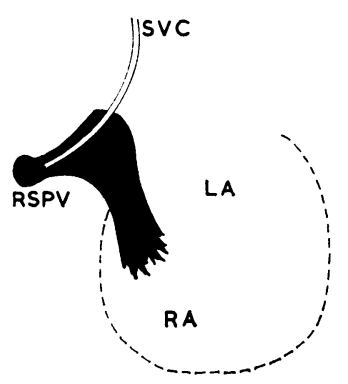

3

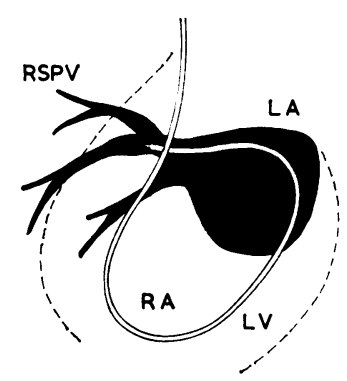

4

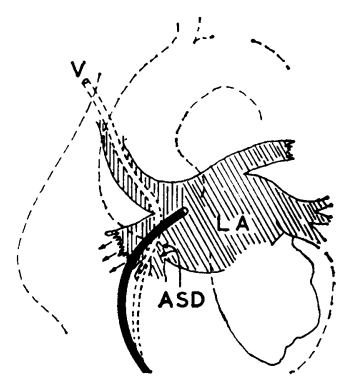

5

FIG. 2.-Cine-angiocardiogram taken with a selective pressure injection into the anomalous vein as the catheter was withdrawn into the SVC. The patient (S.M.) was placed in the left anterior oblique position and the catheter tip in the mouth of the anomalous pulmonary vein. $15 \mathrm{ml}$. of triosil were injected as the catheter was withdrawn into the superior vena cava. Note how the catheter points forward and how all the contrast medium flows into the SVC.

FIG. 3.-Cine-angiocardiogram with selective pressure injection of contrast medium into the anomalous pulmonary vein. The patient was placed in the left anterior oblique position and $15 \mathrm{ml}$. of triosil was injected into the vein about $1 \mathrm{~cm}$. from its site of entry into the SVC. Note how the contrast medium passes backwards into the SVC and RA.

FIG. 4.-Tracing from a cine-angiocardiogram in a patient with an atrial septal defect but normal insertion of the right pulmonary veins into the left atrium. This patient had an ostium primum type of atrial septal defect. The catheter passed backwards first into the left atrium and then forwards into the pulmonary vein. When the cine-angiocardiogram was taken in the left anterior oblique position, the contrast medium flowed forwards into the left atrium, outlining it and the pulmonary veins. Five frames later (film speed 32 frames a second), the right atrium was opacified by flow across the atrial septum.

FIG. 5.-Indirect demonstration of normal pulmonary venous drainage. The atrial septal defect was demonstrated by an injection of contrast medium into the left atrium with the patient in the left anterior oblique position. The pulmonary veins filled by reflux.

The dotted catheter position showed the apparently anomalous course of the pulmonary vein which had a normal drainage route.

complete repair. Clinical examination, the electrocardiogram, and a plain radiograph of the chest are unhelpful since the picture resembles a simple secundum atrial septal defect. Cardiac catheterization too may offer little aid unless the region of the superior vena cava-right atrial junction is explored with the catheter tip and this enters the pulmonary vein. Careful positioning of the patient in the left anterior oblique position will indicate the atrium into which the vein drains. In this position, the atrial septum lies at right angles to the X-ray screen, with the right atrium in front and the left atrium behind. A catheter passing through the atrial septal defect into the left atrium and then into a pulmonary vein with normal drainage will pass backwards first, before passing into the vein. This will not occur if the vein is anomalous and drains into the right atrium or superior vena cava. Selective pulmonary cine-venography, with the patient in the left anterior oblique position will confirm the direction of drainage of the pulmonary veins and provides a permanent visual record. This method is safe provided the catheter tip is withdrawn sufficiently from the point of impaction before the injection of contrast medium is performed.

\section{REFERENCES}

Bahnson, H. T., Spencer, F. C., and Neill, C. A. (1958). Surgical treatment of 35 cases of drainage of pulmonary veins to the right side of the heart. J. thorac. Surg., 36, 777.

Bedford, D. E., Sellors, T. Holmes, Somerville, W., Belcher, J. R., and Besterman, E. M. M. (1957). Atrial septal defect and its surgical treatment. Lancet, 1, 1255.

Brock, R., and Ross, D. N. (1959). The sinus venosus type of atrial septal defect. Guys Hosp. Rep., $108,291$. 
Brotmacher, L., and Fleming, P. (1957). The diagnosis by cardiac catheterization of superior mediastinal anomalous pulmonary veins. Guys Hosp. Rep., 106, 264.

Dow, J. D. (1959). The radiological diagnosis of the sinus venosus type of atrial septal defect. Guys Hosp. Rep., 108, 305 .

Evans, J. R., Rowe, R. D., and Keith, J. D. (1961). The clinical diagnosis of atrial septal defect in children. Amer. J. Med., 30, 345.

Kjellberg, S. R., Mannheimer, E., Rudhe, U., and Jonsson, B. (1959). Diagnosis of Congenital Heart Disease, 2nd ed. Year Book Publishers, Chicago.

Lawrance, K., Grimshaw, V. A., Hoyle, G., Hicks, J. P. N., Nixon, P. F. G., and Wooler, G. H. (1962). Surgical treatment of atrial septal defect and partial anomalous pulmonary venous drainage. J. thorac. Surg., 43, 622 .

LePere, R. H., Thomson, G., and Kohler, C. M. (1962). Anomalous vascular patterns found with atrial septal defect. J. thorac. Surg., 43, 355.

Lewis, F. J., Taufic, M., Varco, R. L., and Niazi, S. (1955). The surgical anatomy of atrial septal defects: Experience with repair under direct vision. Ann. Surg., 142, 401.

Morrow, A. G., Gilbert, J. W., Baker, R. R., and Collins, N. P. (1960). The closure of atrial septal defects utilizing general hypothermia. J. thorac. Surg., 40, 776.

Swan, H. J. C., Kirklin, J. W., Becu, L. M., and Wood, E. H. (1957). Anomalous connection of right pulmonary veins to superior vena cava with interatrial communications. Hemodynamic data in eight cases. Circulation, 16, 54.

Walmsley, R. (1958). The orientation of the heart and the appearance of its chambers in the adult cadaver. Brit. Heart J., 20, 441.

Weidman, W. H., Swan, H. J. C., DuShane, J. W., and Wood, E. H. (1957). A hemodynamic study of atrial septal defect and associated anomalies involving the atrial septum. J. clin. Lab. Med., 50, 165.

Wood, J. C., Conrad, M. E., and Morrow, A. G. (1957). Partial anomalous venous connection. A case report illustrating diagnostic techniques. Amer. Heart J., 54, 422.

Wood, P. (1956). Diseases of the Heart and Circulation, 2nd ed. Eyre and Spottiswoode, London. 